\title{
Influence of Polymer Viscoelasticity on Microscopic Remaining Oil Production
}

\author{
Yiqun Yan ${ }^{1, *}$, Lihui Wang ${ }^{2,3,4, *}$, Guoqiang Sang ${ }^{1}$ and Xu Han ${ }^{1}$ \\ 1 Research Institute of Petroleum Exploration \& Development, PetroChina, Beijing 100083, China; \\ sgqminer@petrochina.com.cn (G.S.); dq_hanxu@hotmail.com (X.H.) \\ 2 Postdoctoral Research Workstation of Daqing Oilfield, Daqing 163458, China \\ 3 Postdoctoral Research Mobile Station of Northeast Petroleum University, Daqing 163318, China \\ 4 Exploration and Development Research Institute of Daqing Oilfield Co., Ltd., Daqing 163712, China \\ * Correspondence: yanyiqun@petrochina.com.cn (Y.Y.); lihuiwang@stu.nepu.edu.cn (L.W.)
}

Citation: Yan, Y.; Wang, L.; Sang, G.; Han, X. Influence of Polymer

Viscoelasticity on Microscopic

Remaining Oil Production. Polymers 2022, 14, 940. https://doi.org/ $10.3390 /$ polym 14050940

Academic Editor: Francesca Lionetto

Received: 4 January 2022

Accepted: 22 February 2022

Published: 26 February 2022

Publisher's Note: MDPI stays neutral with regard to jurisdictional claims in published maps and institutional affiliations.

Copyright: (C) 2022 by the authors. Licensee MDPI, Basel, Switzerland. This article is an open access article distributed under the terms and conditions of the Creative Commons Attribution (CC BY) license (https:// creativecommons.org/licenses/by/ $4.0 /)$.

\begin{abstract}
To investigate the impact of polymer viscoelasticity on microscopic remaining oil production, this study used microscopic oil displacement visualisation technology, numerical simulations in PolyFlow software, and core seepage experiments to study the viscoelasticity of polymers and their elastic effects in porous media. We analysed the forces affecting the microscopic remaining oil in different directions, and the influence of polymer viscoelasticity on the displacement efficiency of microscopic remaining oil. The results demonstrated that the greater the viscosity of the polymer, the greater the deformation and the higher the elasticity proportion. In addition, during the creep recovery experiment at low speed, the polymer solution was mainly viscous, while at high speed it was mainly elastic. When the polymer viscosity reached $125 \mathrm{mPa} \cdot \mathrm{s}$, the core effective permeability reached $100 \times 10^{-3} \mu^{2}$, and the equivalent shear rate exceeded $1000 \mathrm{~s}^{-1}$, the polymer exhibited an elastic effect in the porous medium and the viscosity curve displayed an 'upward' phenomenon. Moreover, the difference in the normal deviatoric stress and horizontal stress acting on the microscopic remaining oil increased exponentially as the viscosity of the polymer increased. The greater the viscosity of the polymer, the greater the remaining oil deformation. During the microscopic visualisation flooding experiment, the viscosity of the polymer, the scope of the mainstream line, and the recovery factor all increased. The scope of spread in the shunt line area significantly increased, but the recovery factor was significantly lower than that in the mainstream line. The amount of remaining oil in the unaffected microscopic area also decreased.
\end{abstract}

Keywords: polymer; viscoelasticity; microscopic remaining oil; force analysis; oil displacement effect

\section{Introduction}

Polymer flooding can greatly improve the oil recovery from waterflooding development oilfields because the higher viscosity of polymer solutions can effectively improve the oil-water mobility ratio, alleviate the conflicts between layers, improve the fluid absorption profile, and enhance displacement [1-3]. Moreover, the viscoelastic effect of the polymer solution can effectively mobilise the remaining oil after waterflooding, improve the oil displacement efficiency, and significantly increase the oil recovery [4-8]. Presently, the Daqing oilfield is the world's largest polymer flooding application block. The annual polymer flooding oil production accounts for more than $30 \%$ of the total oil production. Polymer flooding has great potential for increasing oil production [9-13].

Although polymer flooding has been utilised for more than 40 years, there is still controversy regarding the mechanism of the viscoelastic effect of polymers in the process of percolation [14-18]. The focus of previous studies has been on the impact of polymer viscoelasticity on the oil displacement effect and the critical conditions under which the polymer solution can exhibit elastic effects. When the polymer viscosity is low, the viscoelastic effect cannot occur, and when the viscosity is too high, the viscoelastic effect is 
also impaired. The injection performance and equivalent shear rate in porous media also have significant impacts on viscoelastic performance [19-22]. Therefore, the rational use of the viscoelasticity of a polymer can maximise the oil displacement efficiency at a relatively low polymer dosage. This is of immense significance for the efficient development of oil reservoirs [23-25].

To describe the impact of the polymer viscoelastic effect on the microscopic oil displacement effect more accurately, scholars worldwide have used different methods and theories to study the viscoelasticity of polymers [26-28]. The elastic effect of a compound solution is mainly investigated through experiments that have attracted substantial attention. Gogarty and Levy studied the flow of viscoelastic polymer solutions in a porous medium [29-33]. Several comparison schemes confirmed that the total pressure drop in the flow is affected by the viscosity and elasticity. Furthermore, the Darcy equation has been modified to separately calculate the fluid. The viscosity and elastic pressure drop render this formula suitable for elastic non-Newtonian fluids. Doughty theoretically studied the four constitutive models of Oldroyd, Pao, Bogue, and BKZ to calculate linear rheological parameters. By fitting the rheological experimental data and theoretical calculations, the $\mathrm{BKZ}$ constitutive model has been used to analyse the rheology of the polymer solutions, as described below. Garrouch used artificial glass bead cores and Bailey cores were used for percolation experiments, which proved that a polymer solution is not only viscous but also elastic [34-37]. When using the Debra number to describe viscoelastic flow, a dimensional viscous number has been proposed to describe the viscoelastic flow of a polymer solution, which has been verified by fitting based on experimental data [38]. The above studies have conducted innovative research on the theory of polymer seepage in porous media. However, the analysis of polymer viscoelasticity is inadequate, with only macroscopic theoretical analysis and little experimental verification. The effect of different viscoelastic polymers on microscopic remaining oil production is still unclear, and PolyFlow software has not been used to numerically simulate the force of different viscoelastic polymers on microscopic remaining oil [39]. It is unfeasible to comprehensively analyse the polymer from both macroscopic and microscopic aspects to determine the effects of viscoelasticity on oil displacement.

The purpose of this study was to apply microscopic visualisation oil displacement technology combined with numerical simulation technology and an indoor core seepage experiment to comprehensively analyse the influence of polymer viscoelasticity on the microscopic oil displacement effect. Therefore, we chose the polymer applied in the Daqing oilfield as the research object. Through a creep recovery experiment and an evaluation of rheological properties, the viscosity and elasticity of polymer solutions with different viscosities were quantitatively characterised. A core experiment was used to describe the elastic effect of the polymer solution based on changes in the polymer solution's viscosity in the porous medium. PolyFlow software was used to calculate the normal deviatoric stress and horizontal stress difference of the polymer solutions with different viscosities in simplified micropores. The dynamic images collected by microscopic visualisation of oil displacement technology illustrated the influence of different viscoelastic polymers on the microscopic oil displacement effect.

\section{Materials and Methods}

\subsection{Materials}

Chemicals: Partially hydrolysed polyacrylamide with a molecular weight of 25 million and an effective content of $90 \%$ was produced by Daqing Refining \& Chemical Company (Daqing, China).

Experimental core: The experimental cores included natural cores with a length of $10 \mathrm{~cm}$, diameter of $2.5 \mathrm{~cm}$, and effective permeability of 50,100 , or $150 \times 10^{-3} \mu \mathrm{m}^{2}$.

Microscopic visualisation model of glass etching: Photochemical etching technology was used to create a photoetched glass model. A real pore structure photo of the cast sheet was placed on the glass coated with photosensitive material, and the pore outline pattern 
was copied onto the glass after exposure. The exposed glass template was then treated with hydrofluoric acid to show the impression of the pore structure, and a cover plate was added and sintered at a high temperature. The model, with a size of $40 \mathrm{~mm} \times 40 \mathrm{~mm}$, was developed by a professional company.

Oil and water: Simulated formation water: The $\mathrm{NaCl}$ content of clean water was $950 \mathrm{mg} / \mathrm{L}$, while that of sewage was $4500 \mathrm{mg} / \mathrm{L}$. All systems used clean water to prepare the mother liquor and sewage to dilute the target liquid and model saturated water. Sewage was also used in the displacement experiments. Experimental oil: Simulated oil prepared at a certain ratio between crude oil and kerosene had a viscosity of $10 \mathrm{mPa} \cdot \mathrm{s}$ at $45^{\circ} \mathrm{C}$.

\subsection{Experimental Methodology}

\subsubsection{Core Flow Experiment}

A HAKERS150 rheometer was used to detect the rheology of the polymer solution, and the elastic effect of the polymer in the porous medium was characterised based on the results of the indoor core experiment. Cores with effective permeabilities of 50, 100, and $150 \times 10^{-3} \mu \mathrm{m}^{2}$ were saturated with simulated formation water. The polymer mother liquor was prepared with clear water with an $\mathrm{NaCl}$ content of $950 \mathrm{mg} / \mathrm{L}$. Simulated formation water with an $\mathrm{NaCl}$ content of $4500 \mathrm{mg} / \mathrm{L}$ was used to prepare the target solution, and polymer solutions with viscosities of 40,70, 125, and $215 \mathrm{mPa} \cdot \mathrm{s}$ were prepared. Flow experiments were then initiated for cores with different permeabilities at displacement rates of $8,16,32,64$, and $128 \mathrm{~mL} / \mathrm{h}$. Utilizing this formula, the displacement rate was converted into the shear rate of the polymer solution in the porous medium. At the outlet end, the displacement fluid was used to test its viscosity, and the viscoelastic characteristics of polymer migration in the porous medium were described according to the change in the viscosity of the solution.

\subsubsection{Analysis of Force and Deformation of Microscopic Remaining Oil}

To perform numerical simulation calculations and analyse the flow field, it is necessary to simplify the microchannels with complex shapes in the core. In this study, the flow channel and remaining oil model in the two-dimensional flow field after the flow channel were simplified. The physical model was abstracted and simplified. It was assumed that the displacement fluid flowed between two infinite parallel plates with only one inlet and one outlet. It is assumed that the length of the flow channel in Figure 1 is $\mathrm{L}=100 \mu \mathrm{m}$, the width of the flow channel is $\mathrm{H}=20 \mu \mathrm{m}$, and the remaining oil size is set according to the simulation conditions. The displacement fluid flows under the effect of the pressure difference in the $x$-direction; the effect of gravity is neglected, and the boundary layer is ignored. Presuming that the flow channel is smooth, the interface between the displacement phase and oil is a moving boundary that changes with time. When calculating the microscopic remaining oil deformation, one can assume that three-phase contact points A and B are fixed [40-42].

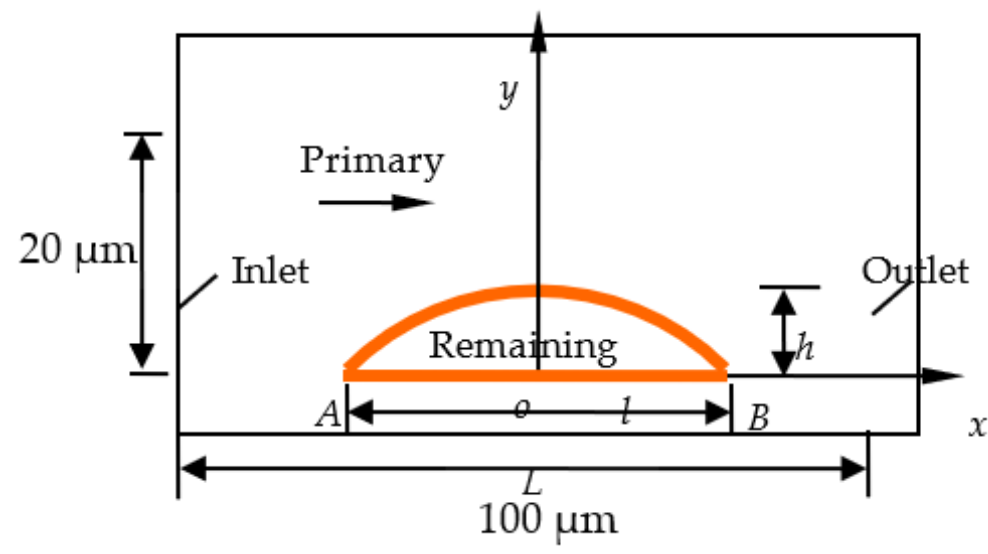

Figure 1. Calculation area diagram. 


\section{Basic equation.}

The continuity equation is an expression of the law of conservation of mass for a system where a fluid is applied to the control body. Its differential form is as follows:

$$
\frac{d \rho}{d t}+\rho d i v u=0
$$

where $\rho$ represents the density of the fluid $\left(\mathrm{g} / \mathrm{cm}^{3}\right)$ and $u$ represents the velocity of the fluid particles $(\mathrm{m} / \mathrm{s})$.

As both the continuous phase (displacement fluid) and dispersed phase (oil) are incompressible fluids, $\rho=\mathrm{C}$. Thus, the continuity equation is deformed as follows:

$$
\nabla \cdot u=0
$$

As the interface is a moving boundary, the effect of interfacial tension is considered in the equation of motion. The equation of motion is expressed as follows:

$$
\frac{\partial(\rho u)}{\partial t}+\nabla \cdot(\rho u)=\nabla \cdot T+\int_{\partial B} d x_{B} \kappa n \sigma \delta\left(x-x_{B}\right)
$$

where $\sigma$ represents the interfacial tension between the polymer solution and the oil $(\mathrm{mN} / \mathrm{m}$; considered as a constant value in this study); $\partial B$ represents the remaining oil surface including point $x_{B} ; \kappa$ represents the curvature of the remaining oil surface; $n$ represents the external method of the remaining oil surface to the unit vector; $\delta\left(x-x_{B}\right)$ represents the second-order $\delta$ function; and T represents the stress tensor [43].

In this study, the upper-satellite Maxwell constitutive equation was used to describe the seepage characteristics of a displacement fluid in simplified pores. The constitutive equation used was as follows:

$$
\tau^{i k}+\lambda \tau^{i k}=\eta_{0} A^{i k}
$$

where $\lambda$ is the relaxation time (s), $\eta_{0}$ is the zero-shear viscosity (Pa.s), $A$ is the first-order Rivlin-Ericksen deformation tensor, and $\tau^{i k}$ is the upper satellite derivative.

In the constitutive equation:

$$
A^{i k}=\sqrt{g^{k k_{g} i i}} \frac{A_{i k}}{\sqrt{g_{k k} g_{i i}}}=A_{i k}=v_{i, j}+v_{j, i}=\frac{\partial v_{i}}{\partial x_{j}}+\frac{\partial v_{j}}{\partial x_{i}}
$$

The upper satellite derivative is:

$$
\tau^{i k}=\frac{\partial \tau^{i k}}{\partial t}+v^{m} \frac{\partial \tau^{i k}}{\partial x^{m}}-\tau^{m k} \frac{\partial v^{i}}{\partial x^{m}}-\tau^{i m} \frac{\partial v^{k}}{\partial x^{m}}
$$

\section{Numerical solution method.}

By solving the flow equation, the stress and horizontal stress difference of the remaining oil can be calculated. When describing the deformation of the microscopic remaining oil, assuming that the remaining oil is in a static state, calculating the flow field of the remaining oil during its displacement by the polymer solution is a steady problem. However, when the remaining oil deforms under the continuous displacement of the polymer solution, it is an unsteady problem, which involves the deformation and reconstruction of the liquid-liquid interface. The polymer solution has complex rheological properties and flow equations. Thus, it is necessary to consider both the interfacial tension and time-varying properties.

As the interface between the displacement fluid and the remaining oil changes dynamically during the displacement process, the deformation of the oil-water interface must be captured by an interface tracking method. In this study, the Lagrange interface-tracking 
method was used to track and reconstruct a two-phase interface. During this method, the interface position of the closed curve is described by parameters. The dynamic interfacial deformation of oil and water changes with the movement of the closed curve. Mark points were set on the closed curve, which were used to track the position change of the interface. Lagrange multipliers can accurately determine the changes in the moving interface and prevent the spread of the value [44].

3. Boundary conditions.

Assuming that the remaining oil is stationary, the calculation of the deviatoric stress and horizontal stress difference acting on the remaining oil is a steady-state problem, and only boundary conditions need to be considered. The inlet and outlet flows were used in this study, and the rest were treated as wall surfaces. The deformation of the remaining oil under the action of a displacement fluid is an unsteady-state problem. At the interface between the displacement fluid and remaining oil, the interface conditions are satisfied, and the tangential stress and velocity are continuous. In this study, it was assumed that the remaining oil and rock contact points A and B do not move. Thus, the remaining microscopic oil does not initially deform.

\subsubsection{Microscopic Visualisation Flooding Experiment}

To study the influence of polymer viscosity on the microscopic oil displacement effect, polymer solutions with different viscosities were prepared to conduct microscopic visualisation experiments, and a Hacker rheometer was used to test the viscosity. The viscosity-concentration relationship is shown in Table 1.

Table 1. Viscosity of polymer solutions with different molecular weights and concentrations.

\begin{tabular}{ccc}
\hline Polymer Molecular Weight & $\begin{array}{c}\text { Polymer Concentration } \\
(\mathbf{m g} / \mathrm{L})\end{array}$ & Viscosity (mPa.s) \\
\hline 25 million & 870 & 40 \\
25 million & 1500 & 70 \\
25 million & 2000 & 125 \\
25 million & 2500 & 215 \\
\hline
\end{tabular}

The polymer solution was configured according to Table 1, and the experimental steps were as follows: (1) The microscopic visualisation model was dried and weighed. Then, a vacuum pump was used to vacuum the saturated simulated oil, the weight of the model after saturation with oil was determined, and the pore volume and initial oil saturation was calculated based on the mass difference before and after the model was saturated with oil. (2) After saturation with oil, the model was placed in a $45{ }^{\circ} \mathrm{C}$ thermostat for $48 \mathrm{~h}$. (3) After removing the model from the thermostat, the water drive was tested first, and the simulated formation water was used for constant rate displacement $(0.03 \mathrm{~mL} / \mathrm{h})$. When the oil was displaced to the outlet end of the model and no oil was produced, the microscopic visual image analysis system was used to calculate the recovery factor in the model at this time. (4) After water flooding, flooding was conducted with the polymer with a viscosity of $40 \mathrm{mPa} \cdot \mathrm{s}$, and no oil was produced after displacement to the outlet. The recovery factor in the model after polymer flooding was then calculated. The polymer concentration viscosity was $70 \mathrm{mPa} \cdot \mathrm{s}$ and the viscosity was $125 \mathrm{mPa} \cdot \mathrm{s}$. The above steps were repeated for oil displacement experiment with a polymer viscosity of $215 \mathrm{mPa} \cdot \mathrm{s}$. (5) After polymer flooding, subsequent water flooding was performed until no oil was produced at the outlet end. The experimental setup is illustrated in Figure 2. 


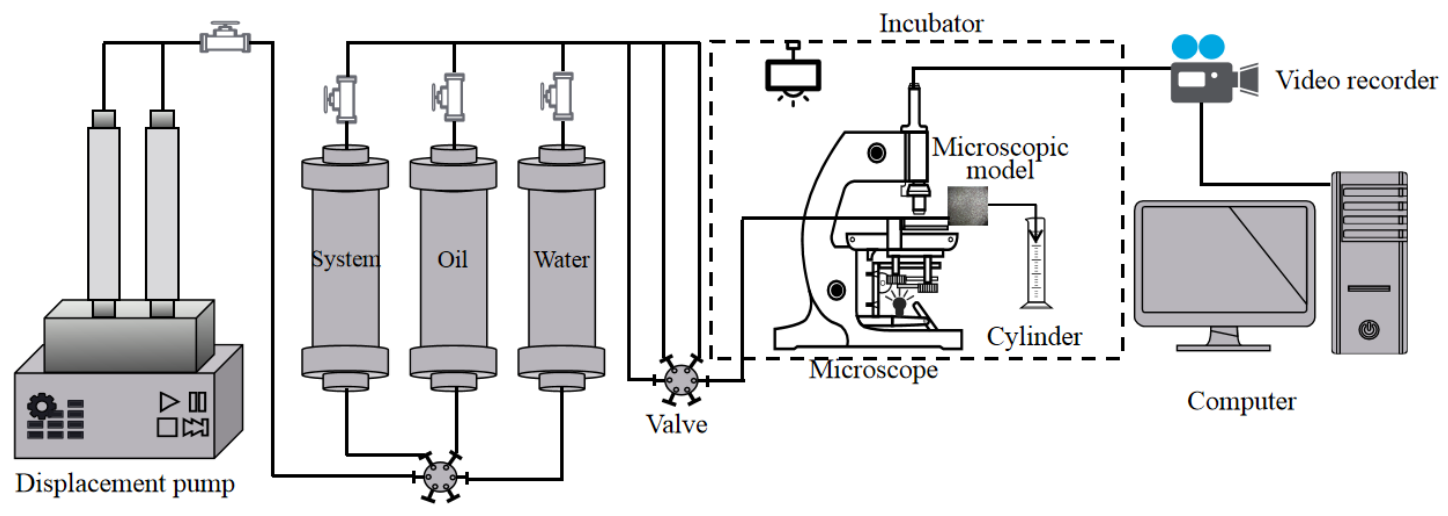

Figure 2. Microscopic visualisation oil displacement device.

\section{Results and Discussion}

\subsection{Analysis of Viscoelasticity of the Polymer Solution}

\subsubsection{Polymer Creep Recovery Performance}

A HAKERS150 rheometer was used to perform dynamic experiments on the creep recovery performance at $45^{\circ} \mathrm{C}$. Changes in the deformation of the polymer solution over time and the changes in viscoelasticity were studied, and the proportions of elasticity and viscosity in the polymer solution were analysed, as shown in Figure 3. In the creep recovery experiment, a constant stress was applied to the sample at $t=t_{0}$. At $t=t_{1}$, the stress was removed, and the strain changed with time during the process. In the creep stage, the applied stress produces a transient response, the viscoelasticity of each substance has a combined effect, and their specific contributions cannot be clearly separated [45]. After the applied stress was released, it entered the recovery state. The advantage of the recovery phase is that the percentage of the total strain can be decomposed into a permanently maintained viscous part and recoverable elastic part. The ratio of the strain contributed by the elastic part and that contributed by the viscous part to the total strain reflect the elasticity and viscosity of the viscoelastic solution, respectively. In the creep phase, the deformation increased with time. In the recovery phase, as the time increased, the deformation first decreased sharply and then stabilised. As the viscosity of the polymer solution increased, the proportion of the elastic part increased.

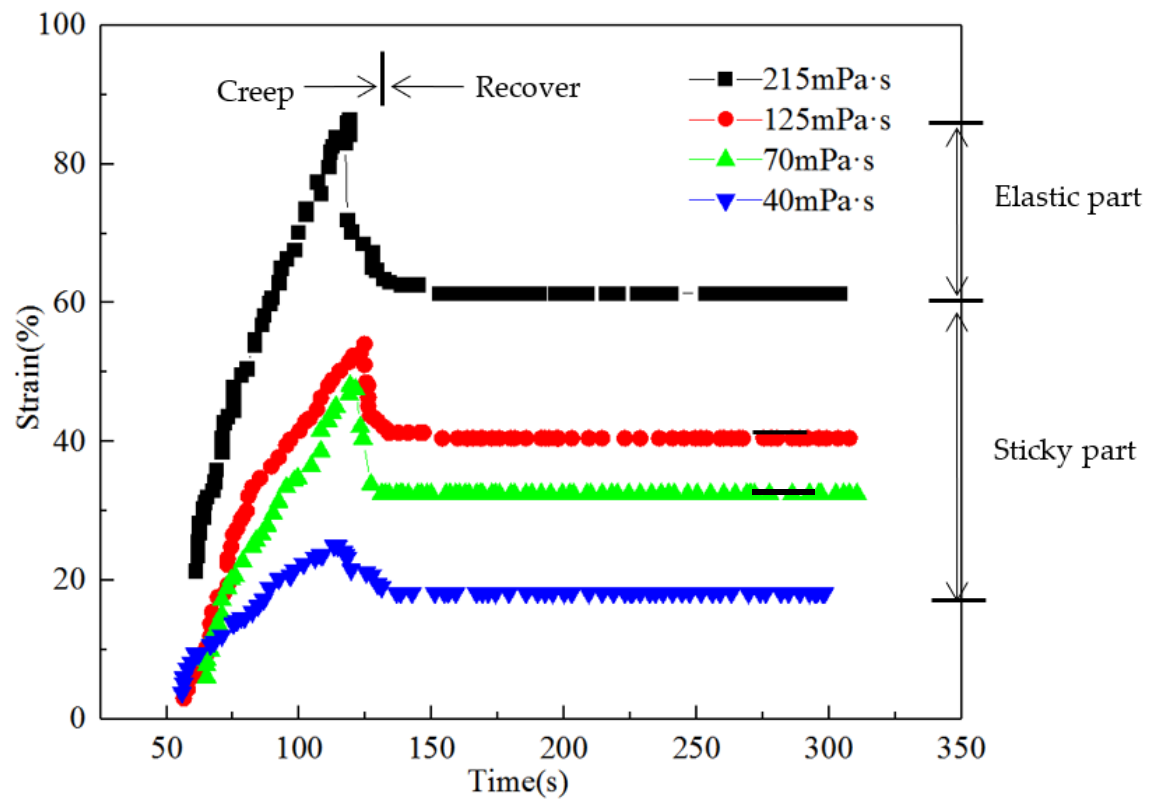

Figure 3. Creep recovery curves of polymer solutions with different viscosities. 


\subsubsection{Quantitative Characterisation of Polymer Viscoelasticity}

As shown from the change curve of the storage modulus $\left(G^{\prime}\right)$ and dissipation modulus $\left(G^{\prime \prime}\right)$ of polymer solutions with different viscosities with angular velocity, as the angular velocity increased, the storage modulus and energy dissipation modulus increased. In addition, as the viscosity of the solution increased, the storage modulus and energy dissipation modulus increased, the number of molecules per unit volume increased, and the ability of molecules to attract and entangle with each other increased. This led to an increase in the strength of viscoelasticity. The storage and dissipation moduli of each viscous polymer solution exhibited an intersection. The intersection point moved to the left; $G^{\prime \prime}$ was greater than $G^{\prime}$ before the intersection point, and $G^{\prime}$ was greater than $G^{\prime \prime}$ after the intersection point. At low angular velocities, the polymer solutions were mainly viscous, whereas at high angular velocities, they were mainly elastic [46]. As shown in Figure 4.

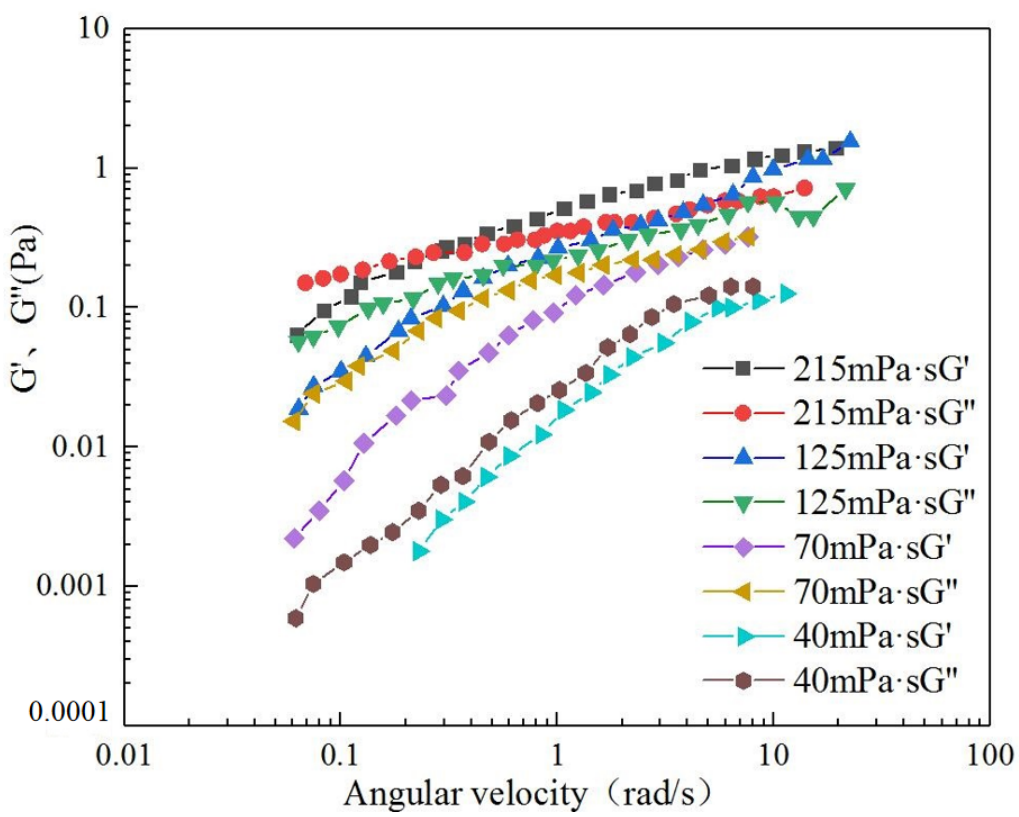

Figure 4. Changes in the storage and energy dissipation moduli of polymers with different viscosities.

Figure 5 shows the storage and energy dissipation moduli of polymer solutions with viscosities of 40,70,125, and $215 \mathrm{mPa} \cdot \mathrm{s}$ at shear rates of 0.06283 and $6.283 \mathrm{~s}^{-1}$. According to the results of the dynamic experiment, when the shear rate was low $\left(0.06283 \mathrm{~s}^{-1}\right)$, the proportions of the elastic and viscous components remained relatively unchanged at $44.75 \%$ and $54.98 \%$, respectively. Under the formation shear rate $\left(6.283 \mathrm{~s}^{-1}\right)$, the proportion of elasticity far exceeded that of the viscous part, and with an increase in the mass concentration of the solution, the proportion of the elastic part increased. However, the magnitude of the increase gradually decreased. This phenomenon indicates that the elasticity of the solution is not negligible when the polymer solution flows into the porous medium. The greater the viscosity of the polymer solution, the more significant the elastic effect. The results of the creep recovery experiment under low stress conditions and the dynamic experiment under low shear rate conditions were slightly different because the two measurement methods are based on different principles. In the creep recovery experiment, the elastic and viscous components were expressed by the relative magnitude of the contribution of the elastic and viscous properties to the strain. The ratio of the strain contributed by the elastic properties and that contributed by the viscous properties to the total strain directly reflects the elasticity and viscosity of the viscoelastic solution. The storage and dissipation moduli were used to characterise the viscoelasticity of the solution based on the relationship between the force and corresponding deformation. When the solution viscosity reached $125 \mathrm{mPa} \cdot \mathrm{s}$, the results of the two methods were consistent [47]. 


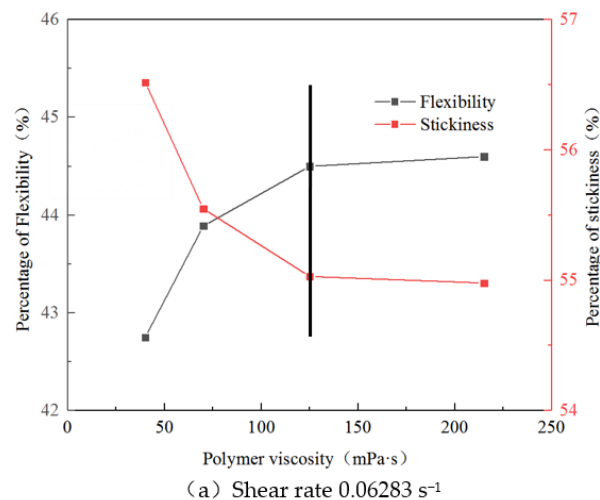

(a) Shear rate $0.06283 \mathrm{~s}^{-1}$

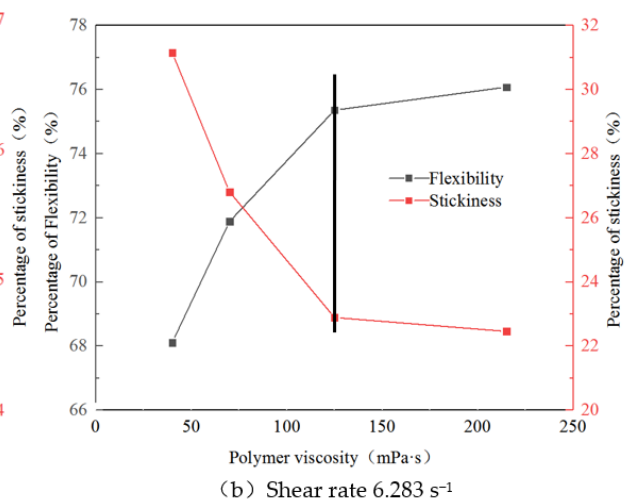

(b) Shear rate $6.283 \mathrm{~s}^{-1}$

Figure 5. Proportion of viscoelasticity under different shear rates and concentrations. (a) Shear rate $0.06283 \mathrm{~s}^{-1}$. (b) Shear rate $6.283 \mathrm{~s}^{-1}$.

To understand the structure of partially hydrolysed polyacrylamide, the morphology of the film formed by titrating the above polymer solution onto a glass slide was observed and analysed using an atomic force microscope. Figure 6 shows the structural morphology of the polymer solutions with different viscosities; the scanning range was $20 \mu \mathrm{m} \times 20 \mu \mathrm{m}$. Although the polymer segments had a certain probability of collision in the solution, they could not be stably bonded by van der Waals forces owing to the action of the solvent and thermal movement. After the polymer solution was dropped onto the surface of the glass slide, the polyacrylamide molecules moved freely to form the most stable distribution. The distribution of polyacrylamide was fixed until the water evaporated. Polyacrylamide does not stretch and advance individually during movement, although multiple molecules can gather into small particles to move. From the atomic force scanning images of polymer solutions with different viscosities, when the viscosity was $40 \mathrm{mPa} \cdot \mathrm{s}$, the particles were almost isolated and did not have an orderly distribution, indicating that the viscoelasticity of the solution was very low. When the viscosity was $70 \mathrm{mPa} \cdot \mathrm{s}$, the distance between particles decreased and aggregates formed; however, the distribution of the aggregates was uneven, and the distance between them was still relatively large. Compared to those in a polymer solution with a viscosity of $40 \mathrm{mPa} \cdot \mathrm{s}$, the number of molecules increased, and the viscoelasticity increased. When the viscosity increased to $125 \mathrm{mPa} \cdot \mathrm{s}$, an ordered arrangement of polyacrylamide polymer chains easily formed, and a relatively regular network structure developed between the particles. The structure of the solution at this point was ideal and the proportion of elasticity reached a maximum. When the viscosity of the solution continued to increase to $215 \mathrm{mPa} \cdot \mathrm{s}$, the probability of intermolecular collisions increased; thus, a self-similar tree structure formed between particles, and the increase in the number of molecules led to an increase in the overall viscoelasticity of the solution [48].

\subsubsection{Elastic Effect of Polymers in Porous Media}

When a polymer flows into a pore, it is subjected to shearing and stretching by the porous medium, and the polymer solution exhibits viscoelasticity when the viscosity and shear rate reach a certain level. The different displacement rates allow the calculation of the shear rate of the polymer solution during its formation. The viscosity curves of polymer solutions with different viscosities after shearing in core samples with different permeabilities are shown in Figure 7. In the core with a permeability of $50 \times 10^{-3} \mu \mathrm{m}^{2}$, as the shear rate increased, the viscosity of the polymer solutions with different viscosities gradually decreased and did not exhibit viscoelasticity. This is mainly because the permeability and porosity were low, while the shear rate of the polymer solution in the core was high, which significantly damaged the polymer molecular structure. Consequently, the viscosity of the polymer gradually decreased, thus not exhibiting viscoelasticity. In the core with a permeability of $100 \times 10^{-3} \mu^{2}$, when the polymer viscosity was 40 and $70 \mathrm{mPa} \cdot \mathrm{s}$, as the shear rate increased, the viscosity gradually decreased. When the viscosity of the polymer 
solution reached $125 \mathrm{mPa} \cdot \mathrm{s}$ and the shear rate exceeded $1000 \mathrm{~s}^{-1}$, the polymer solution after core shearing exhibited viscoelasticity, and the viscosity curve exhibited an 'upward' trend.

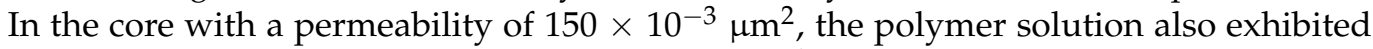
viscoelasticity when the shear rate reached $1000 \mathrm{~s}^{-1}$ and the polymer viscosity exceeded $125 \mathrm{mPa} \cdot \mathrm{s}$, and the slope of the viscosity curve was greater than that of the core with a permeability of $100 \times 10^{-3} \mu^{2}$. Based on the above results, under the current polymertype conditions, when the polymer viscosity reached $125 \mathrm{mPa} \cdot \mathrm{s}$, the equivalent shear rate reached $1000 \mathrm{~s}^{-1}$. When the effective permeability of the core reached $100 \times 10^{-3} \mu \mathrm{m}^{2}$, the polymer solution exhibited an elastic effect in the porous medium [48].

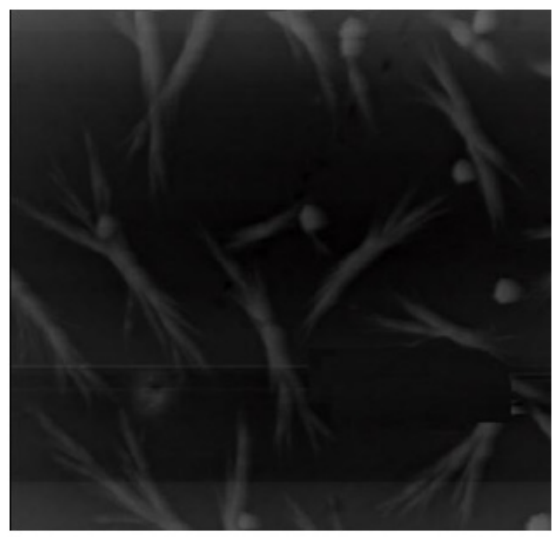

$40 \mathrm{mPa} \cdot \mathrm{s}$

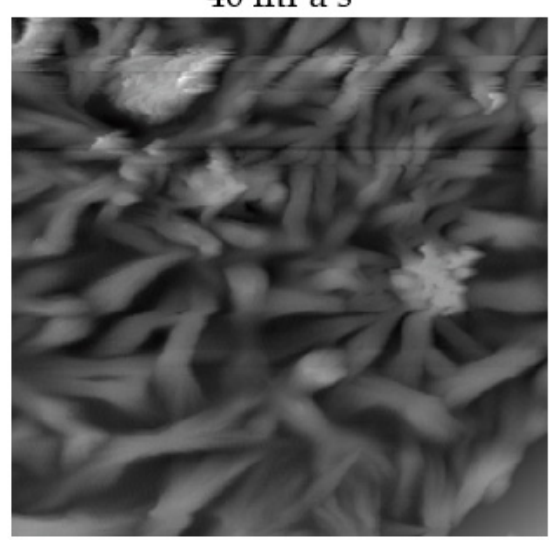

$125 \mathrm{mPa} \cdot \mathrm{s}$

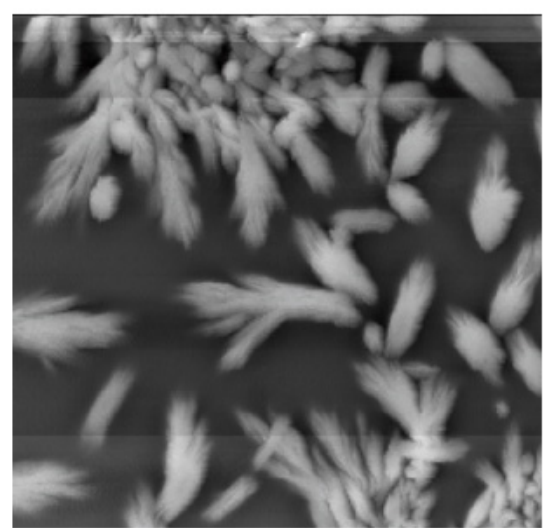

$70 \mathrm{mPa} \cdot \mathrm{s}$

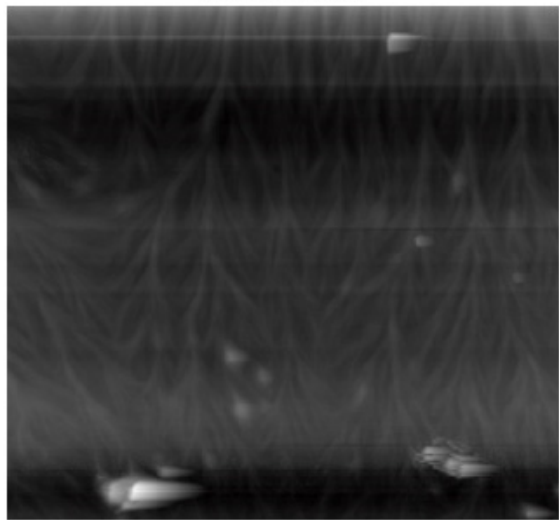

$215 \mathrm{mPa} \cdot \mathrm{s}$

Figure 6. Structure and morphology of polymer solutions with different viscosities $(20 \mu \mathrm{m} \times 20 \mu \mathrm{m})$.

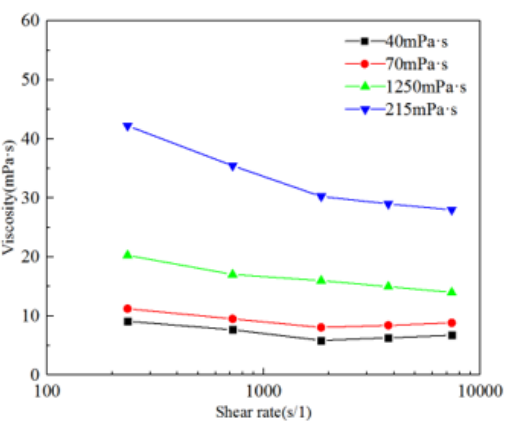

(a) $50 \times 10^{-3} \mu \mathrm{m}^{2}$

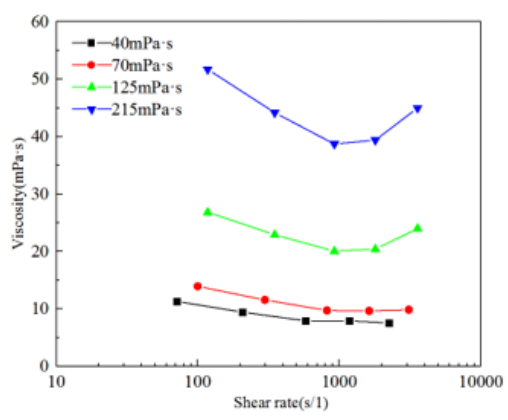

(b) $100 \times 10^{-3} \mu \mathrm{m}^{2}$

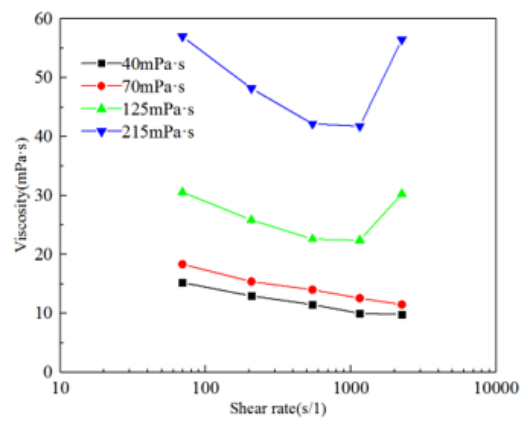

(c) $150 \times 10^{-3} \mu \mathrm{m}^{2}$

Figure 7. Core viscosity changes at different permeabilities. (a) $50 \times 10^{-3} \mu \mathrm{m}^{2}$. (b) $1.00 \times 10^{-3} \mu \mathrm{m}^{2}$. (c) $1.50 \times 10^{-3} \mu^{2}$. 


\subsection{Force Analysis of Microscopic Remaining Oil}

\subsubsection{Normal Deviator Stress on Microscopic Remaining Oil}

To analyse the microscopic forces acting on various polymers passing through the remaining oil, PolyFlow software (ANSYS, Inc., Canonsburg, PA, USA) was used to calculate the pressure gradient in microchannels of $0.02 \mathrm{MPa} / \mathrm{m}$, Newtonian fluid (oil) with a viscosity of $10 \mathrm{mPa} \cdot \mathrm{s}$, and polymers with different viscosities. The stress field was calculated for the flow of the elastic fluid through the remaining oil, as shown in Figure 8. In this study, the normal stress was positive and negative, the pressure was positive, and the tension was negative. The tangential stress was positive along the flow direction and negative against the flow direction. Horizontal and vertical stress was positive in the coordinate axis direction, and negative in the opposite direction. As the viscosity of the displacement fluid increased, the stress acting on the remaining oil also increased. When the viscosity doubled, the stress on the remaining oil almost doubled. Normal stress is the force perpendicular to the surface of the remaining oil [49]. This force causes the remaining oil to bulge or sag in the normal direction, which increases its local deformation. The remaining upstream oil is compressed, and the remaining downstream oil is stretched. Tangential deviator stress is the force along the tangential direction of the residual oil. The greater the force, the greater the angular deformation. Tangential stress is the greatest in the middle of the remaining oil, illustrating an inverted parabolic distribution law. The horizontal stress describes the changing law of stress along the direction of flow. The horizontal stress experienced by the remaining oil is positive, and thus, it deforms along the direction of flow. Upstream of the remaining oil, the vertical stress is downward, and the downstream vertical stress is upward, also reflecting the deformation process of the remaining oil after it is stressed.

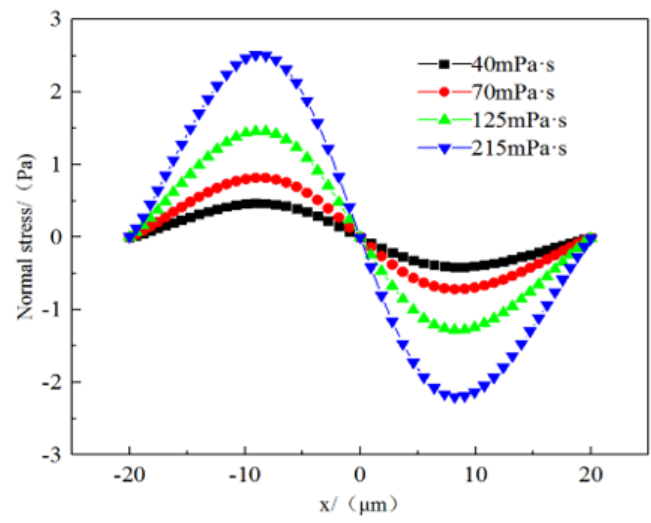

(a) Normal stress curve

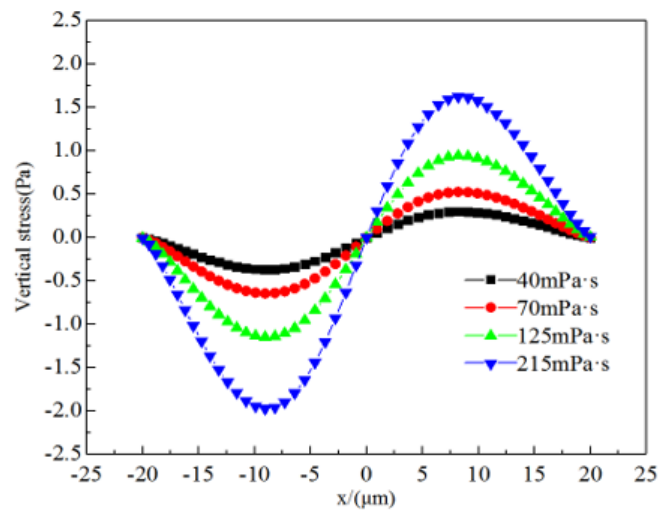

(c) Vertical stress curve

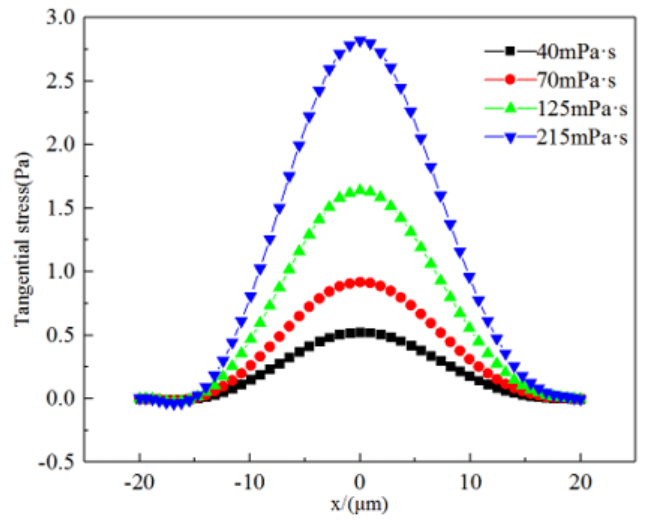

(b) Tangential stress curve

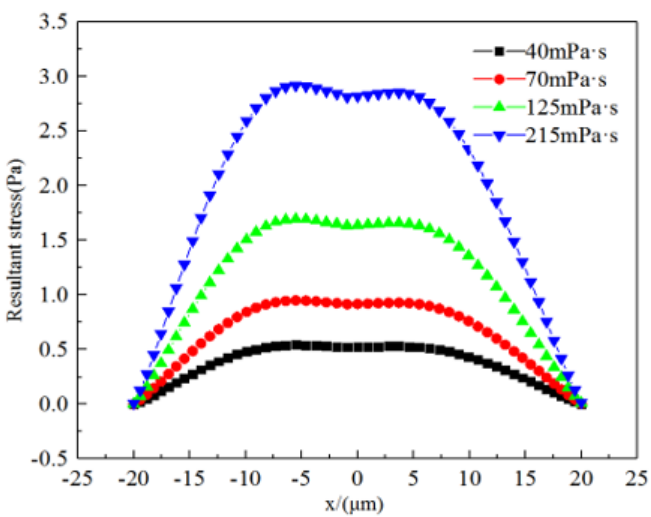

(d) Total stress curve

Figure 8. Stress curves. (a) Normal stress curve. (b) Tangential stress curve. (c) Vertical stress curve. (d) Total stress curve. 


\subsubsection{Horizontal Stress Differences on Microscopic Remaining Oil}

The above calculation results for different types of stress only present the difference in the stress distribution of the remaining oil under different viscosities and cannot intuitively provide the difference in the displacement force acting on the remaining oil. The force that truly reflects the displacement effect of the displacement fluid on the remaining oil is the difference in the horizontal stress acting on the same horizontal line of the remaining oil interface, that is, the horizontal stress difference, as shown in Figure 9. This figure presents the change in the horizontal stress experienced by the remaining oil under the action of displacement fluids with different viscosities. The horizontal stress difference reflects the magnitude of stress of the displacement fluid acting on the remaining oil at different positions along the flow direction. When the viscosity of the displacement fluid increased, the horizontal stress difference also increased. When the viscosity doubled, the horizontal stress difference also doubled. When displacement fluids with different viscosities flowed through the remaining oil, shearing and stretching forces were generated, such that the distribution of remaining oil changed, and the wetting angle also changed accordingly, resulting in wetting hysteresis. Along the flow direction, the rear part of the remaining oil was pushed downstream towards the wall, producing a negative curvature, while the front part of the remaining oil tended to roll along the wall. If the interfacial tension between the rock and remaining oil was high, the remaining oil would eventually break, with part of it separating from the mother liquid droplet, and part of it continuing to adhere to the wall $[50,51]$.

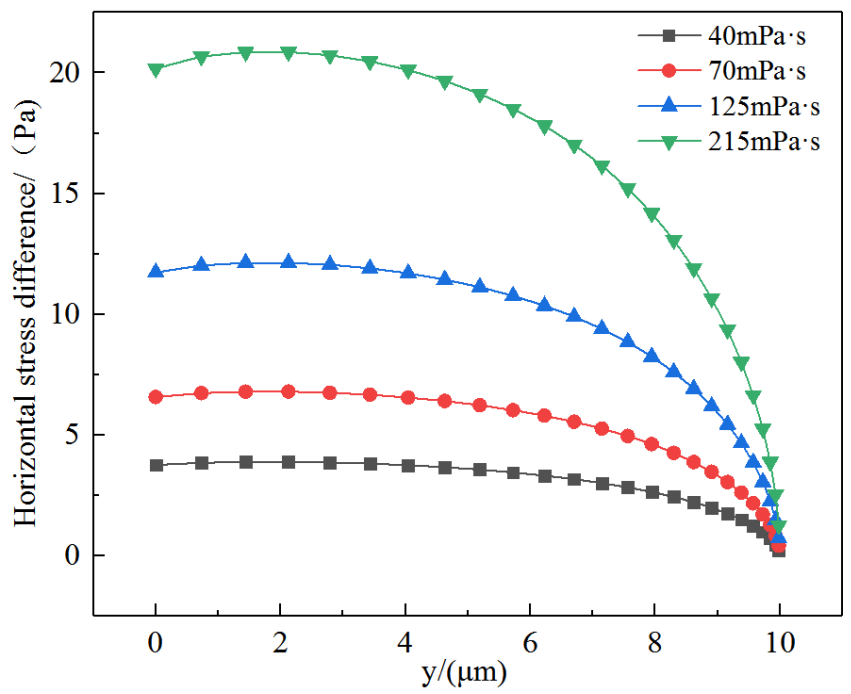

Figure 9. Variation of horizontal stress differences.

\subsection{Microscopic Visualization of Oil Displacement}

\subsubsection{Influence of Polymer Viscosity on the Microscopic Oil Displacement Effect}

The arrows in the microscopic visualisation model in Figure 10 represent the displacement direction. Polymer solutions with different viscosities were prepared for the micro-displacement experiments. After water flooding, the total amount of residual oil significantly decreased. However, a large amount of residual oil remained in the model. After waterflooding, polymer flooding was performed using polymers with different viscosities, and the large-area clusters retained after waterflooding were divided into multiple small-area clusters of the remaining oil. As the viscosity of the polymer solution increased, the recovery factor of the model continued to increase. When the viscosity of the polymer reached $215 \mathrm{mPa} \cdot \mathrm{s}$, the recovery factor in the microscopic visualisation model was high, but a large amount of microscopic oil was still retained. 


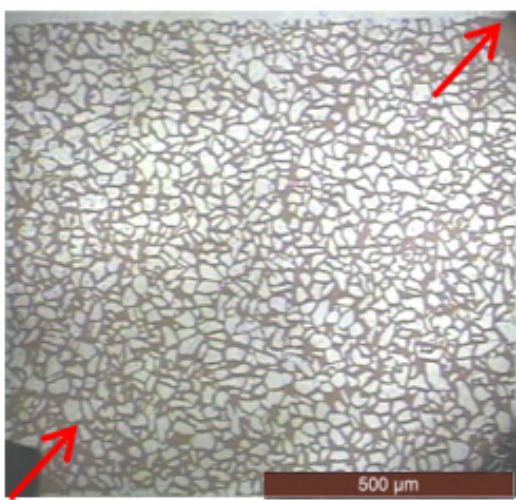

(a) Saturated oil

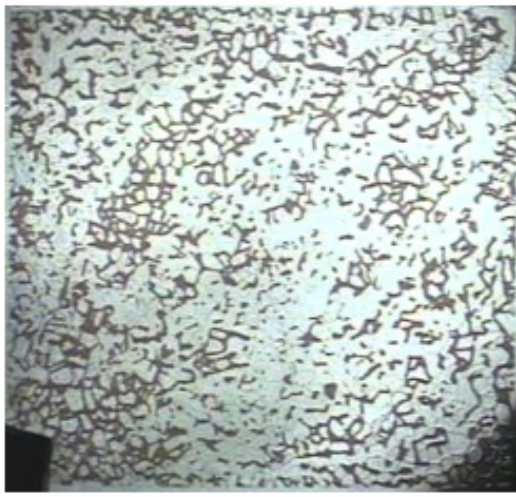

(d) $70 \mathrm{mPa} \cdot \mathrm{s}$

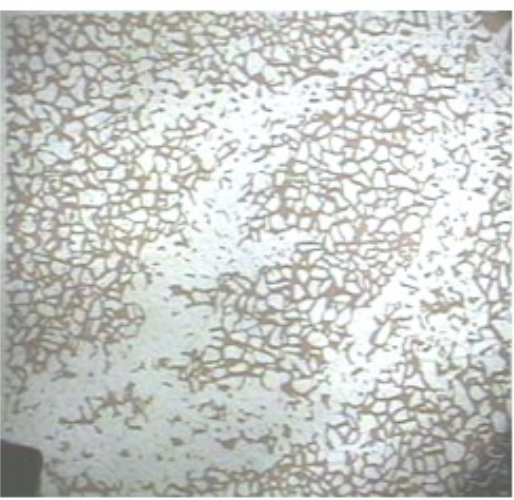

(b) Waterflooding

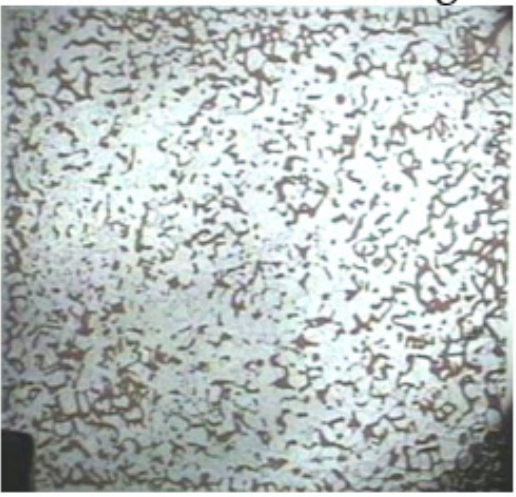

(e) $125 \mathrm{mPa} \cdot \mathrm{s}$

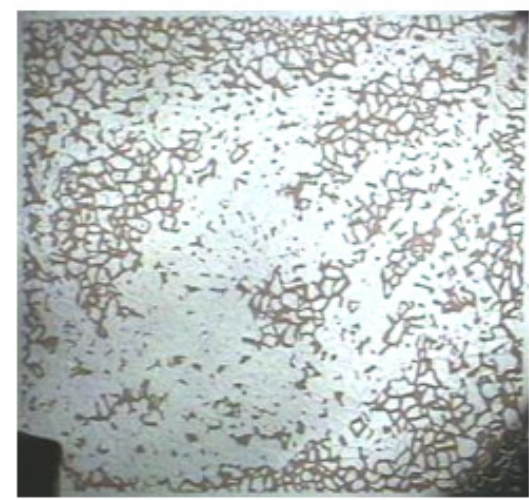

(c) $40 \mathrm{mPa} \cdot \mathrm{s}$

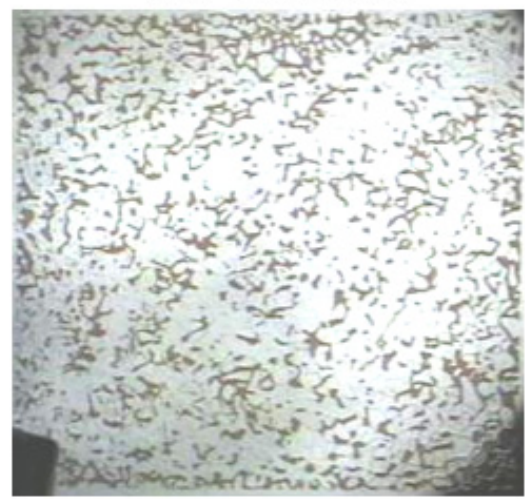

(f) $215 \mathrm{mPa} \cdot \mathrm{s}$

Figure 10. Oil displacement effect of polymers with different viscosities. (a) Saturated oil. (b) Waterflooding. (c) $40 \mathrm{mPa} \cdot \mathrm{s}$. (d) $70 \mathrm{mPa} \cdot \mathrm{s}$. (e) $125 \mathrm{mPa} \cdot \mathrm{s}$. (f) $215 \mathrm{mPa} \cdot \mathrm{s}$.

A microscopic visual image analysis system was used to calculate the recovery factor during different displacement stages, and the results are listed in Table 2. As the viscosity of the polymer increased, its viscoelasticity became more obvious, and its contribution to the oil displacement efficiency increased significantly. The microscopic remaining oil production in the model increased and the recovery factor increased. When the polymer viscosity was $215 \mathrm{mPa} \cdot \mathrm{s}$, the final recovery factor was $71.682 \%$, which was $40.61 \%$ higher than that during waterflooding, and $28.318 \%$ of the remaining oil in the model could not be removed.

Table 2. Recovery efficiency from flooding with polymer with different viscosities.

\begin{tabular}{cccc}
\hline Viscosity (mPa.s) & $\begin{array}{c}\text { Polymer Flooding } \\
\text { Recovery Factor (\%) }\end{array}$ & $\begin{array}{c}\text { Ultimate Recovery } \\
\mathbf{( \% )}\end{array}$ & $\begin{array}{c}\text { Enhanced Value of } \\
\text { Polymer Flooding } \\
\text { Recovery Factor (\%) }\end{array}$ \\
\hline Water drive & 31.072 & 31.072 & - \\
40 & 58.503 & 58.503 & 27.431 \\
70 & 59.675 & 65.563 & 34.491 \\
125 & 58.663 & 66.996 & 35.924 \\
215 & 60.353 & 71.682 & 40.610 \\
\hline
\end{tabular}

\subsubsection{Microscopic Remaining Oil Production at Different Locations}

To specifically analyse the utilisation of polymers with different viscosities on the microscopic remaining oil at different locations, the visualisation model was divided into nine regions, and the nine regions were further divided into three types: main flow lines, diversion lines, and microscopic unaffected areas. The main flow line included Areas 3, 5 , and 7, and the diversion line included Areas 2, 4, 6, and 8. The microscopically unaffected 
areas included Areas 1 and 9. The oil recovery factor for different area types was then calculated, as shown in Figure 11.

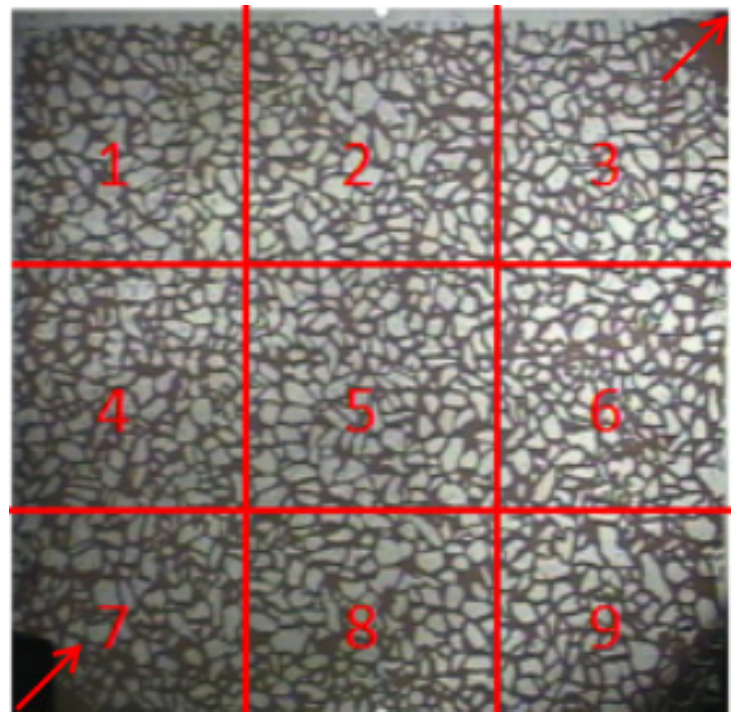

Figure 11. Regional division of the microscopic visualisation model.

\section{Mainstream area.}

A comparative analysis of the production of microscopic residual oil in the main flow line during flooding with polymers with different viscosities is shown in Figure 12. Area 7 represents the injection end. When the displacement phase viscosity was low, the displacement pressure difference was small, the fluidity control ability was weak, and the driving force was less than the seepage resistance. The injected water protruded toward the production end along the dominant seepage channel and advanced forward in a non-piston form in the model. Fingering and circumvention pose serious problems; in this area, many clusters of residual oil formed because of circumvention and other reasons. As the viscosity of the displacement phase increased gradually, the viscoelasticity increased, resistance increased during the seepage process, the displacement pressure difference increased, and the spread range expanded. The partially retained clusters of the remaining oil changed from large clusters to scattered small clusters. When the polymer viscosity reached $215 \mathrm{mPa} \cdot \mathrm{s}$, the remaining oil volume in the microscopic visualisation model significantly decreased. However, as the seepage distance increased, the polymer fluidity control ability weakened, and bypass and fingering still occurred. The remaining oil volume in the main flow line from the injection end to the production end gradually increased.

A microscopic visualisation oil displacement image analysis system was used to calculate the remaining oil area in different areas on the mainstream line, and the recovery factor at different stages was calculated through the changes in the microscopic remaining oil in the field of view. The results are presented in Table 3. In the main flow line area, under the same viscosity conditions, as the seepage distance increased, the increase in oil recovery gradually decreased from the injection end to the production end. In the same area, as the viscosity of the polymer increased, the viscoelastic effect enhanced and the recovery factor gradually improved. 


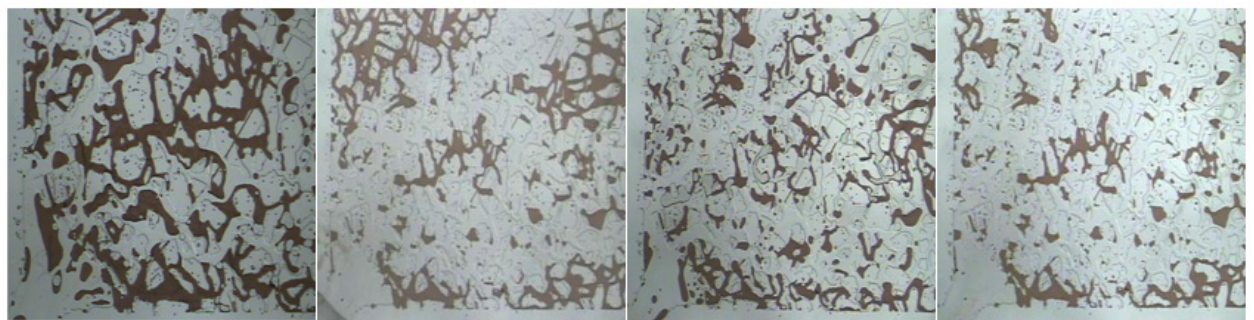

(a) Area 7

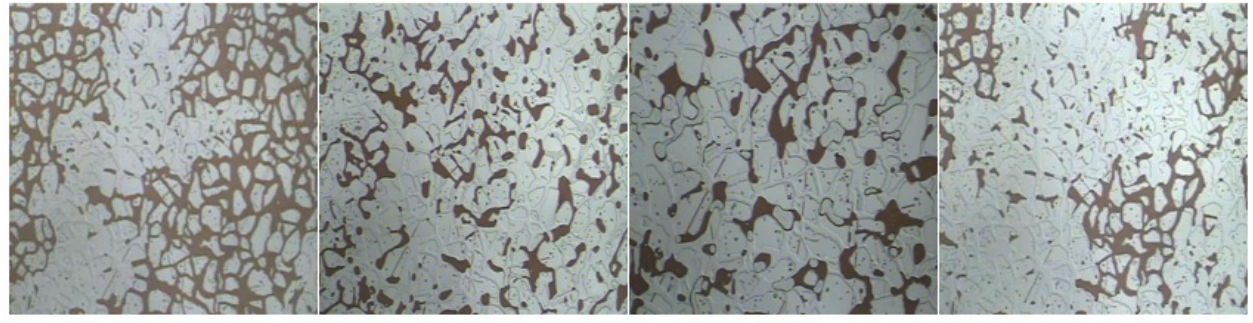

(b) Area 5

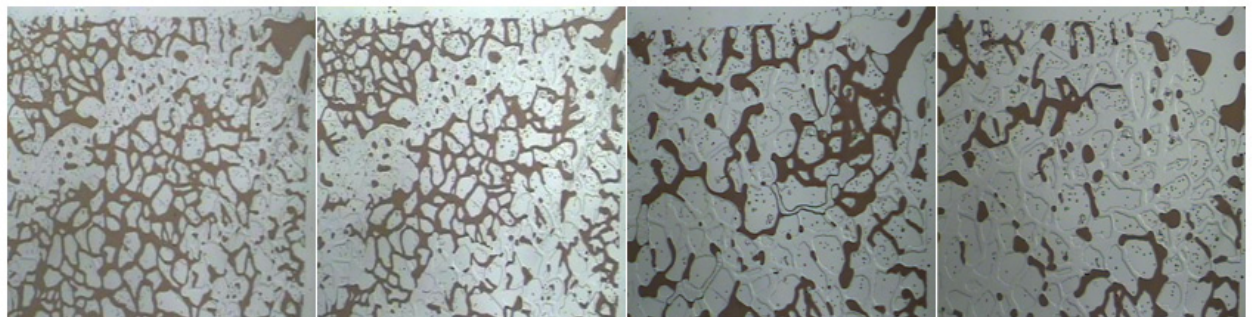

(c) Area 3

$40 \mathrm{mPa} \cdot \mathrm{s}$

$70 \mathrm{mPa} \cdot \mathrm{s}$

$125 \mathrm{mPa} \cdot \mathrm{s}$

$215 \mathrm{mPa} \cdot \mathrm{s}$

Figure 12. Microscopic remaining oil production in the mainstream area under different viscosity conditions. (a) Area 7. (b) Area 5. (c) Area 3.

Table 3. Changes in recovery factors in different areas of the mainstream line.

\begin{tabular}{ccccc}
\hline \multirow{2}{*}{ Position } & \multicolumn{4}{c}{ Recovery Efficiency of Polymer Flooding at Different Viscosities (\%) } \\
\cline { 2 - 5 } & $\mathbf{4 0 ~} \mathbf{~ P a} \cdot \mathbf{s}$ & $\mathbf{7 0 ~} \mathbf{~ P a} \cdot \mathbf{s}$ & $\mathbf{1 2 5} \mathbf{~} \mathbf{P a} \cdot \mathbf{s}$ & $\mathbf{2 1 5} \mathbf{~} \mathbf{P a} \cdot \mathbf{s}$ \\
\hline Area 7 & 51.25 & 59.64 & 68.26 & 72.23 \\
Area 5 & 44.20 & 58.04 & 64.05 & 70.19 \\
Area 3 & 37.02 & 50.78 & 59.65 & 63.84 \\
\hline
\end{tabular}

\section{Diversion line area.}

After flooding with polymers with different viscosities, the remaining oil in the model had a significant difference in the displacement effect on the mainstream line, and the distribution characteristics of the microscopic remaining oil on the diversion line were also significantly different. The remaining oil production at the injection end, Areas 8 and 4, and at the production end, Areas 6 and 2, were compared and analysed, as shown in Figure 13. When the polymer viscosity was $40 \mathrm{mPa} \cdot \mathrm{s}$, owing to the low viscosity of the displacement phase and the small driving force, the solution migrated along large pores with little resistance against forming a dominant seepage channel. As the injection volume increased, the remaining oil in the diversion line area no longer changed. As the viscosity of the displacement phase increased, the range of spread increased, effectively suppressing the fingering phenomenon. Not only did the remaining oil production on the mainstream line increase but the remaining oil on the diversion line also significantly decreased. After polymer flooding, oil still remained in the diversion line area, and the potential to tap this microscopic remaining oil was still relatively high. 


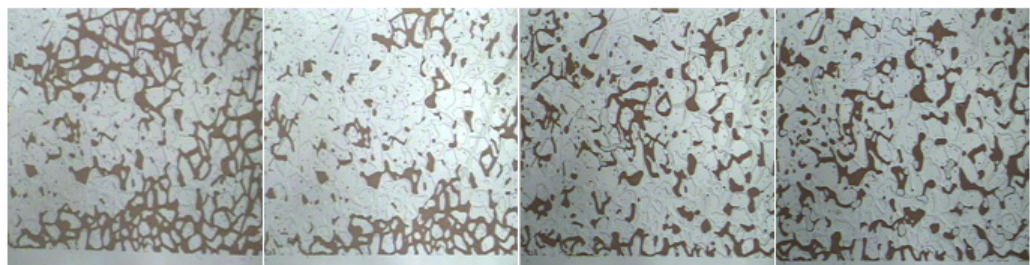

(a) Area 8

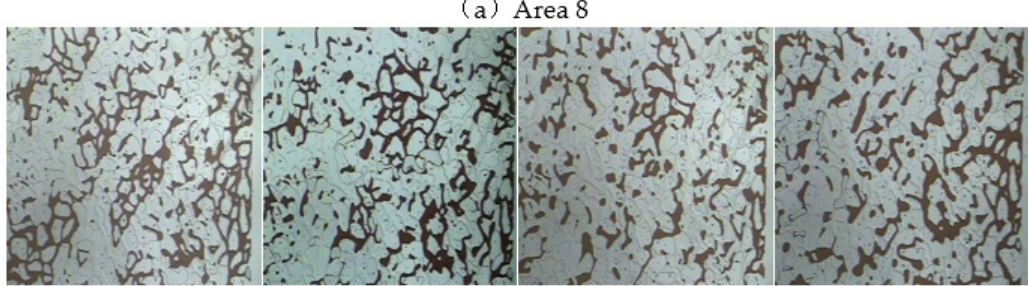

(b) Area 6

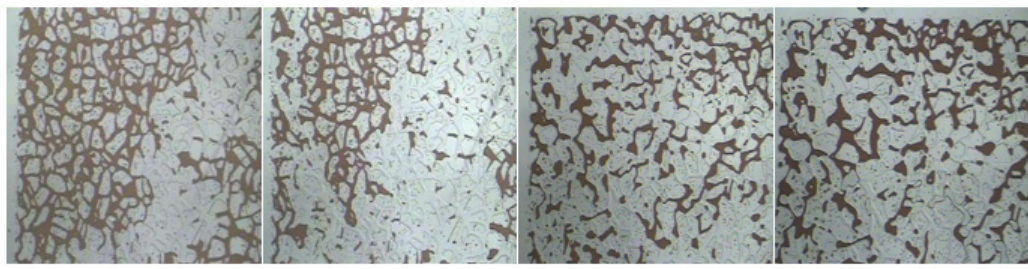

(c) Area 4

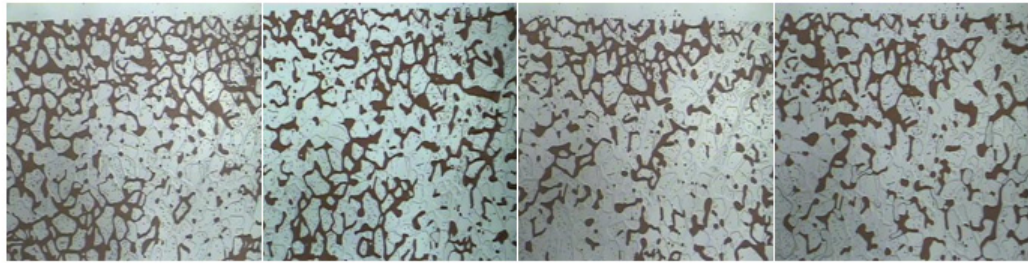

$40 \mathrm{mPa} \cdot \mathrm{s} \quad 70 \mathrm{mPa} \cdot \mathrm{s} \quad 125 \mathrm{mPa} \cdot \mathrm{s} \quad 215 \mathrm{mPa} \cdot \mathrm{s}$

Figure 13. Microscopic remaining oil production in the shunt line area under different viscosity conditions. (a) Area 8. (b) Area 6. (c) Area 4. (d) Area 2.

A microscopic visual oil displacement image analysis system was used to calculate changes in the recovery factor in different areas of the shunt line. The results are presented in Table 4 . As Areas 8 and 4 were closer to the injection end, the recovery factor was higher. However, owing to the differences in the range of polymer spread, the recovery factor in the shunt line area was significantly lower than that in the mainstream area.

Table 4. Changes in the recovery factor in different areas of the diversion line.

\begin{tabular}{|c|c|c|c|c|}
\hline \multirow{2}{*}{ Position } & \multicolumn{4}{|c|}{ Recovery Efficiency of Polymer Flooding with Different Viscosities (\%) } \\
\hline & $40 \mathrm{mPa} \cdot \mathrm{s}$ & $70 \mathrm{mPa} \cdot \mathrm{s}$ & $125 \mathrm{mPa} \cdot \mathrm{s}$ & $215 \mathrm{mPa} \cdot \mathrm{s}$ \\
\hline Area 8 & 23.17 & 25.95 & 35.16 & 41.19 \\
\hline Area 6 & 15.73 & 25.40 & 34.52 & 45.28 \\
\hline Area 4 & 22.35 & 24.50 & 29.42 & 37.50 \\
\hline Area 2 & 11.57 & 16.58 & 20.73 & 25.87 \\
\hline
\end{tabular}

3. Microscopic unaffected area.

In the microscopic visualisation model area division, Areas 1 and 9 are at the edges of the model, and the remaining oil in these areas is difficult to produce, as shown in Figure 14 When the viscosity was low, the displacement fluid had a lower degree of production for the remaining oil in these two areas, mainly as these two areas were perpendicular to the displacement direction, and the displacement force in the seepage direction could not effectively spread to these areas. As a result, the remaining oil in Areas 1 and 9 near 
the edges of the model was not extracted, and only a small amount of the remaining oil was carried away near the edge of the diversion line area. As the viscosity of the polymer gradually increased, the amount of oil remaining in the microscopically unaffected area significantly decreased. The main reason for this is that, as the viscosity of the displacement phase increases, the resistance increases during the seepage process, the displacement pressure difference increases, and the scope of the polymer solution significantly expands during the displacement process. The viscoelastic effect of the polymer solution can increase its seepage capacity in the porous medium and the production of remaining oil from these two areas. The residual oil in the unaffected microscopic area at the edge of the model thus significantly decreased.

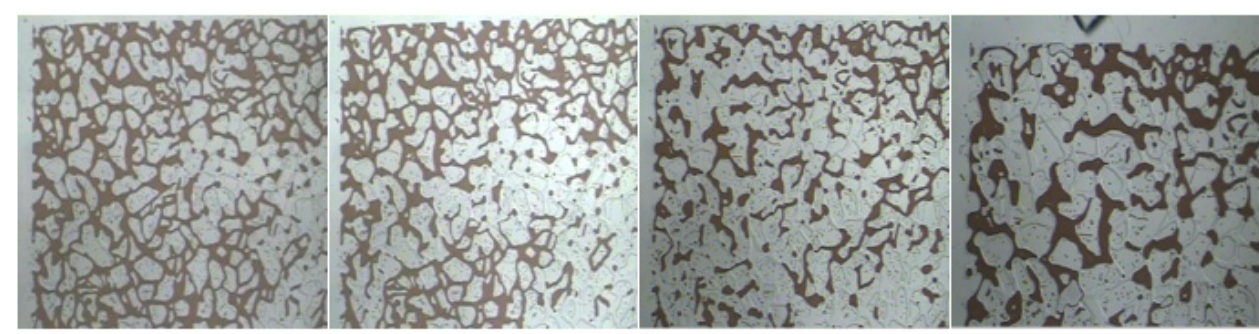

(a) Area 1

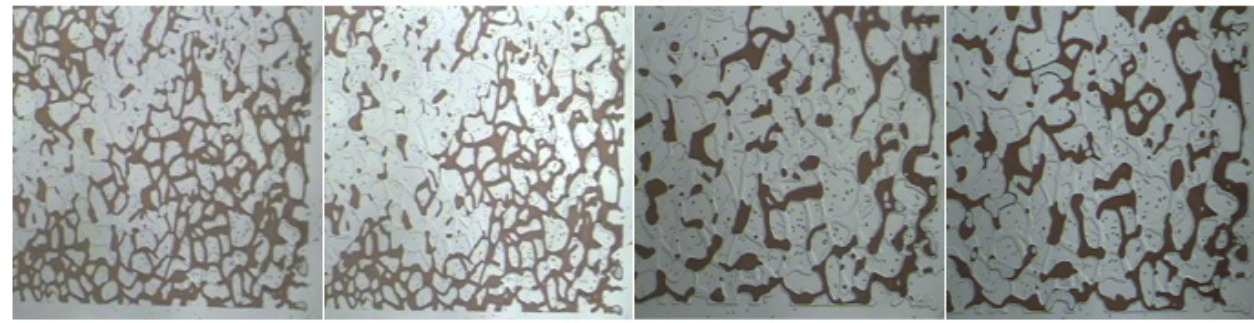

$$
40 \mathrm{mPa} \cdot \mathrm{s} \quad 70 \mathrm{mPa} \cdot \mathrm{s} \quad 215 \mathrm{mPa} \cdot \mathrm{s}
$$

Figure 14. Distribution characteristics of remaining oil in the microscopic unaffected area after polymer flooding. (a) Area 1. (b) Area 9.

A microscopic visual oil displacement image analysis system was used to calculate the recovery factor for different viscosities in the unaffected microscopic area. The results are presented in Table 5. The proportion of remaining oil in the microscopically unaffected area after flooding with polymers with different viscosities decreased. When the polymer viscosity was $215 \mathrm{mPa} \cdot \mathrm{s}$, the recovery factors were only $21.05 \%$ and $20.31 \%$, indicating that the remaining oil in the microscopic unaffected area was relatively low and that there was still a large amount of retained oil.

Table 5. Variations in recovery in the microscopic unaffected area.

\begin{tabular}{ccccc}
\hline \multirow{2}{*}{ Position } & \multicolumn{4}{c}{ Recovery Efficiency of Polymer Flooding with Different Viscosities (\%) } \\
\cline { 2 - 5 } & $\mathbf{4 0} \mathbf{~} \mathbf{~ P a} \cdot \mathbf{s}$ & $\mathbf{7 0 ~} \mathbf{~ P a} \cdot \mathbf{s}$ & $\mathbf{1 2 5} \mathbf{~} \mathbf{P a} \cdot \mathbf{s}$ & $\mathbf{2 1 5} \mathbf{~} \mathbf{P a} \cdot \mathbf{s}$ \\
\hline Area 1 & 25.66 & 24.12 & 23.75 & 21.05 \\
Area 9 & 24.03 & 23.09 & 22.70 & 20.31 \\
\hline
\end{tabular}

\section{Conclusions}

(1) As per the creep recovery experiment, the greater the viscosity of the polymer solution, the greater the proportion of elasticity. Atomic force microscope images demonstrated that when the polymer concentration was low, the particles were almost isolated and did not form an ordered distribution, resulting in a low viscoelasticity of the solution. As the polymer concentration increased, the distance between particles decreased and aggregates formed. Nevertheless, the distribution of aggregates was not uniform, 
and the distance between them was relatively large. When the viscosity increased to $125 \mathrm{mPa} \cdot \mathrm{s}$, an orderly arrangement of polymer chain segments formed, and a relatively regular network structure formed between particles. The structure of the solution was ideal at this point, and the proportion of elasticity reached its maximum.

(2) When the polymer viscosity reached $125 \mathrm{mPa} \cdot \mathrm{s}$, the equivalent shear rate reached $1000 \mathrm{~s}^{-1}$, the core effective permeability reached $100 \times 10^{-3} \mu \mathrm{m}^{2}$, the polymer solution exhibited viscoelasticity in the porous medium, and the viscosity curve exhibited an 'upward' phenomenon.

(3) Based on the calculations from the numerical simulations using PolyFlow software, as the viscosity of the polymer increased, the stress acting on the remaining oil increased. When the viscosity doubled, the stress on the oil film also doubled. As displacement fluids of different viscosities flowed through the remaining oil, differences occurred in the normal and horizontal stresses, which deformed the remaining oil and changed the wetting angle accordingly.

(4) In the microscopic visualisation flooding experiment, as the viscosity of the polymer increased, the degree of production of the remaining oil in the model gradually increased. Under the same viscosity conditions on the main flow line, as the seepage distance increased, the recovery factor gradually decreased from the injection to the production end. In the same area, as the viscosity of the polymer increased, the recovery factor gradually increased. Increasing the viscosity of the polymer significantly increased the sweep range in the shunt line area, but the recovery factor was significantly lower than that of the mainstream line. Increasing the viscosity of the polymer also affected the remaining oil in the microscopic unaffected area. When the polymer viscosity was $215 \mathrm{mPa} \cdot \mathrm{s}$, the recovery factors were only $21.05 \%$ and $20.31 \%$, indicating that the remaining oil in the microscopic unaffected area after polymer flooding was relatively low, with a substantial amount of oil still being retained.

Author Contributions: Conceptualization, Y.Y. and L.W.; methodology, G.S.; software, X.H.; validation, Y.Y.; formal analysis, X.H.; investigation, L.W.; resources, L.W.; data curation, L.W.; writingoriginal draft preparation, L.W.; writing—review and editing, G.S.; visualization, X.H.; supervision, Y.Y.; project administration, Y.Y.; funding acquisition, X.H. All authors have read and agreed to the published version of the manuscript.

Funding: This research received no external funding.

Institutional Review Board Statement: Not applicable.

Informed Consent Statement: Not applicable.

Data Availability Statement: Not applicable.

Acknowledgments: The authors would like to acknowledge the financial support from the China Postdoctoral Science Foundation (No. 2021M693509). The authors would also like to acknowledge the State Key Laboratory of Enhance Oil Recovery, Beijing 10083, China for providing crude oil and equipment for experiments.

Conflicts of Interest: We would like to submit the enclosed manuscript titled "Influence of Polymer Viscoelasticity on Microscopic Remaining Oil Production," for publication in Polymers. No conflict of interest exists regarding the submission of this manuscript, which has been approved by all authors for publication. I would like to declare on behalf of my coauthors that the research described is original, has not been published previously, and is not under consideration for publication elsewhere, in whole or in part. All listed authors approved the enclosed manuscript.

\section{References}

1. Pearson, D.S. Recent advances in the molecular aspects of polymer viscoelasticity. Rubber Chem. Technol. 1987, 60, 439-496. [CrossRef]

2. Yang, Y.; Tao, L.; Iglauer, S. Quantitative statistical evaluation of micro residual oil after polymer flooding based on X-ray micro computed-tomography scanning. Energy Fuels 2020, 34, 10762-10772. [CrossRef] 
3. Lotfollahi, M.; Koh, H.; Li, Z. Mechanistic simulation of residual oil saturation in viscoelastic polymer floods. In Proceedings of the SPE EOR Conference at Oil and Gas West Asia, Muscat, Oman, 21-23 March 2016. [CrossRef]

4. Vermolen, E.C.; Haasterecht, M.J.; Masalmeh, S.K. A systematic study of the polymer visco-elastic effect on residual oil saturation by core flooding, SPE EOR Conference at Oil and GasWest Asia. OnePetro 2014, 17, 225-236. [CrossRef]

5. Santamaria, O.; Lopera, S.H.; Riazi, M. Phenomenological study of the micro-and macroscopic mechanisms during polymer flooding with $\mathrm{SiO}_{2}$ nanoparticles. J. Pet. Sci. Eng. 2021, 198, 108135. [CrossRef]

6. Zhong, H.; Li, Y.; Zhang, W. Microflow mechanism of oil displacement by viscoelastic hydrophobically associating water-soluble polymers in enhanced oil recovery. Polymers 2018, 10, 628. [CrossRef]

7. Peng, Q.; Lashgari, H.; Luo, H. Simulation of Viscoelastic Polymer Flooding-From the Lab to the Field. In Proceedings of the SPE Annual Technical Conference and Exhibition, Dallas, TX, USA, 24-26 September 2018. [CrossRef]

8. Xia, H.; Wang, D.; Zhang, J. Quantitative description of contribution of elasticity of polymer solution to oil displacement efficiency. J. China Univ. Pet. 2012, 4, 1-10. [CrossRef]

9. Rock, A.; Hincapie, R.E.; Tahir, M. On the Role of Polymer Viscoelasticity in Enhanced Oil Recovery: Extensive Laboratory Data and Review. Polymers 2020, 12, 2276. [CrossRef] [PubMed]

10. Yue, M.; Zhu, W.; Han, H. Experimental research on remaining oil distribution and recovery performances after nano-micron polymer particles injection by direct visualization. Fuel 2018, 212, 506-514. [CrossRef]

11. Irfan, M.; Stephen, K.D.; Lenn, C.P. An experimental study to investigate novel physical mechanisms that enhance viscoelastic polymer flooding and further increase desaturation of residual oil saturation. Upstream Oil Gas Technol. 2021, 6, 100026. [CrossRef]

12. Sharafi, M.S.; Jamialahmadi, M.; Hoseinpour, S.A. Modeling of viscoelastic polymer flooding in Core-scale for prediction of oil recovery using numerical approach. J. Mol. Liq. 2018, 250, 295-306. [CrossRef]

13. Rahimi, S.; Habibian, M.; Salehi, M.B. Effect of polymer molar mass and montmorillonite content on polymer flooding using a glass micromodel. Appl. Clay Sci. 2018, 163, 186-195. [CrossRef]

14. Yang, G.F.; Wang, G.; Gao, J.-S.; Xu, C.-M. Mechanism for enhancing oil-displacement efficiency by betaine surfactant after polymer flooding. Acta Pet. Sin. 2007, 28, 86.

15. Urbissinova, T.S.; Kuru, E. Effect of elasticity during viscoelastic polymer flooding: A possible mechanism of increasing the sweep efficiency. J. Can. Pet. Technol. 2010, 49, 49-56. [CrossRef]

16. Pal, N.; Mandal, A. Enhanced oil recovery performance of gemini surfactant-stabilized nanoemulsions functionalized with partially hydrolyzed polymer/silica nanoparticles. Chem. Eng. Sci. 2020, 226, 115887. [CrossRef]

17. Qi, M.; Wegner, J.; Falco, L. Pore-scale simulation of viscoelastic polymer flow using a stabilised finite element method. In Proceedings of the SPE Reservoir Characterization and Simulation Conference and Exhibition, Abu Dhabi, United Arab Emirates, 16-18 September 2013. [CrossRef]

18. Tang, E.G.; Yin, H.J.; Zhong, H.Y. Flow Characteristics of Viscoelastic Polymer Solution in Contraction Micro-Pores of Different Pore-Throat Ratio. Adv. Mater. Res. 2012, 598, 370-373. [CrossRef]

19. Zhang, M.; Kang, W.; Yang, H.; Zhou, B.; Li, Z.; He, Y.; Grigory Yurievich, K.; Alexander Viktorovich, L. De-emulsification performance and mechanism of $\beta-C D$ reverse demulsifier for amphiphilic polymer oil in water (O/W) emulsion. J. Mol. Liq. 2021, 342, 1-7. [CrossRef]

20. Zhong, H.; Tian, Z.; Yin, H. Flow of viscoelastic polymer solutions through a planar contraction with a boundary layer effect. Chem. Technol. Fuels Oils 2012, 48, 393-402. [CrossRef]

21. Qiannan, Y.; Liu, Y.K.; Song, L. Experimental study on surface-active polymer flooding for enhanced oil recovery: A case study of Daqing placanticline oilfield, NE China. Pet. Explor. Dev. 2019, 46, 1206-1217. [CrossRef]

22. Ameli, F.; Moghbeli, M.R.; Alashkar, A. On the effect of salinity and nano-particles on polymer flooding in a heterogeneous porous media: Experimental and modeling approaches. J. Pet. Sci. Eng. 2019, 174, 1152-1168. [CrossRef]

23. Kumar, N.; Mandal, A. Oil-in-water nanoemulsion stabilized by polymeric surfactant: Characterization and properties evaluation for enhanced oil recovery. Eur. Polym. J. 2018, 109, 265-276. [CrossRef]

24. Wang, P.; Ma, W.; Zhang, Y. Relationship between the Molecular Coil Dimension and the Energy Storage Modulus of Polymer Solution Configured with Oilfield-Produced Sewage. Int. J. Anal. Chemistry 2020, 2020, 1-8. [CrossRef] [PubMed]

25. Zhong, C.; Meng, X.; Deng, J. Steady shear and viscoelastic properties of a micro-crosslinked acrylamide-based terpolymer. $J$. Appl. Polym. Sci. 2011, 120, 666-675. [CrossRef]

26. Xia, H.; Wang, L.; Han, P. Microscopic Residual Oil Distribution Characteristics and Quantitative Characterization of Producing Degree Based on Core Fluorescence Analysis Technology. Geofluids 2021, 2021, 8827721 . [CrossRef]

27. Hou, J.; Li, Z.; Zhang, S. Computerized tomography study of the microscopic flow mechanism of polymer flooding. Transp. Porous Media 2009, 79, 407-418. [CrossRef]

28. Xie, C.; Xu, K.; Mohanty, K. Nonwetting droplet oscillation and displacement by viscoelastic fluids. Phys. Rev. Fluids 2020, 5, 063301. [CrossRef]

29. Clarke, A.; Howe, A.M.; Mitchell, J. Mechanism of anomalously increased oil displacement with aqueous viscoelastic polymer solutions. Soft Matter 2015, 11, 3536-3541. [CrossRef]

30. Clarke, A.; Howe, A.M.; Mitchell, J. How viscoelastic-polymer flooding enhances displacement efficiency. SPE J. 2016, 21, 06750687. [CrossRef] 
31. Wever, D.A.Z.; Picchioni, F.; Broekhuis, A.A. Comblike polyacrylamides as flooding agent in enhanced oil recovery. Ind. Eng. Chem. Res. 2013, 52, 16352-16363. [CrossRef]

32. Han, X.; Wang, L.; Xia, H. Mechanism underlying initiation of migration of film-like residual oil. J. Dispers. Sci. Technol. 2021, 1, 1-21. [CrossRef]

33. Liu, L.L.; Wang, L.H.; Zhang, Y. Mechanical Analysis of Stress Acted on Residual Oil Film by Polymer Solution. Xinjiang Pet. Geol. 2010, 31, 533. [CrossRef]

34. Jian, Q.L.; Ming, Z.W.; Bao, J.B. Review of transport mechanisms and numerical simulation studies of preformed particle gel for conformance control. J. Pet. Sci. Eng. 2012, 206, 109051. [CrossRef]

35. Khalilinezhad, S.S.; Mohammadi, A.H.; Hashemi, A. Rheological characteristics and flow dynamics of polymer nanohybrids in enhancing oil recovery from low permeable carbonate oil reservoirs. J. Pet. Sci. Eng. 2021, 197, 107959. [CrossRef]

36. Cao, B.; Luo, P. Experiment Study on Viscoelasticity of Associative Polymer Solutions. J. Southwest Pet. Univ. 2007, 5, 572-577.

37. Han, X.; Chen, Z.; Zhang, X. Surfactant-polymer flooding formulated with commercial surfactants and enhanced by negative salinity gradient. Fuel 2020, 274, 117874. [CrossRef]

38. Wei, P.; Pu, W.; Sun, L. Role of water-soluble polymer on foam-injection process for enhancing oil recovery. J. Ind. Eng. Chem. 2018, 65, 280-289. [CrossRef]

39. Zhen, S.; Guo, C.; Gang, S. A new mathematical model for polymer flooding. Acta Pet. Sin. 2008, 29, 409-413. [CrossRef]

40. Jian, Q.L.; Ming, Z.W.; Bao, J.B.; Seright, R.S.; Zhang, Y.; Cercone, D.; Samson, N. Impact of Rheology Models on Horizontal Well Polymer Flooding in a Heavy Oil Reservoir on Alaska North Slope: A Simulation Study. In Proceedings of the Offshore Technology Conference, Virtual, Houston, TX, USA, 16-19 August 2021. [CrossRef]

41. Sayadi, S.; Chahardowli, M.; Simjoo, M. Study of Mechanisms Affecting the Heavy Oil Recovery in the Polymer Alternating $\mathrm{CO}_{2}$ Injection using Micro-model. J. Pet. Res. 2021, 31, 83-95. [CrossRef]

42. Yao, Y.; Ming, Z.W.; Bao, J.B. Descriptive statistical analysis of experimental data for wettability alteration with surfactants in carbonate reservoirs. Fuel 2021, 310, 122110. [CrossRef]

43. Ding, L.; Wu, Q.; Zhang, L. Application of fractional flow theory for analytical modeling of surfactant flooding, polymer flooding, and surfactant/polymer flooding for chemical enhanced oil recovery. Water 2020, 12, 2195. [CrossRef]

44. Han, X.; Kurnia, I.; Chen, Z. Effect of oil reactivity on salinity profile design during alkaline-surfactant-polymer flooding. Fuel 2019, 254, 115738. [CrossRef]

45. Wang, L.; Xia, H.; Han, P.; Cao, R. Synthesis of New PPG and Study of Heterogeneous Combination Flooding Systems. J. Dispers. Sci. Technol. 2020, 2, 1-15. [CrossRef]

46. Kurnia, I.; Zhang, G.; Han, X. Zwitterionic-anionic surfactant mixture for chemical enhanced oil recovery without alkali. Fuel 2020, 259, 116236. [CrossRef]

47. Yao, Y.; Ming, Z.W.; Wang, L.K. A review of wettability alteration using surfactants in carbonate reservoirs. Adv. Colloid Interface Sci. 2021, 294, 102477. [CrossRef] [PubMed]

48. Chen, Z.; Han, X.; Kurnia, I. Adoption of phase behavior tests and negative salinity gradient concept to optimize Daqing oilfield alkaline-surfactant-polymer flooding. Fuel 2018, 232, 71-80. [CrossRef]

49. Zhou, R.N.; Zhang, D.; Wei, J.G. Experiment on the profile control effect of different strength gel systems in heterogeneous reservoir. Energy Rep. 2021, 7, 6023-6030. [CrossRef]

50. Hu, Y.; Zhao, Z.; Dong, H.; Mikhailova, M.D.; Davarpanah, A. Hybrid Application of Nanoparticles and Polymer in Enhanced Oil Recovery Processes. Polymers 2021, 13, 1414. [CrossRef]

51. Tahir, M.; Hincapie, R.E.; Langanke, N.; Ganzer, L.; Jadger, P. Coupling Microfluidics Data with Core Flooding Experiments to Understand Sulfonated/Polymer Water Injection. Polymers 2020, 12, 1227. [CrossRef] 